\title{
Meloxicam Decreases the Formation of Peritoneal Adhesions in an Experimental Surgical Model in Rats
}

Luis Alfredo Hernandez Villarroel' ${ }^{1}$ Henry Fernandez' ${ }^{1}$ Luisa Cesin ${ }^{1}$

\begin{abstract}
Background: Inflammatory adhesions result from an inflammatory response of the peritoneum during an intra-abdominal inflammatory process secondary to thermal or mechanical injury, infection, radiation, ischemia, dissection, abrasion or foreign body reaction. Adhesions produce consequences such as: infertility, intestinal obstruction, and pelvic-abdominal pain. The objective of this study is to evaluate the effects of Meloxicam, a selective cyclooxygenase-2 inhibitor, on the formation of postoperative peritoneal adhesions in an experimental animal model. Methods: Twenty female Wistar rats were submitted to laparotomy. Postoperative peritoneal adhesions were induced by scorching the serous surface of the colon. The animals were randomly divided into two experimental groups: one group received Meloxicam intramuscularly for 7 days, and the other served as a control group. They were sacrificed and evaluated at 15 days. Results: In the animals given Meloxicam, it was observed that a decrease in number $(p=0.018)$, severity $(p=0.004)$, extension $(p=0.011)$, density $(p=0.023)$, degree of inflammation $(p=0.002)$, vascular proliferation $(p=0.004)$ and fibrosis $(p=0.029)$ of adhesions, compared to the control group. Conclusion: In conclusion, this study demonstrated that the administration of Meloxicam intramuscularly significantly decreases the formation of postoperative peritoneal adhesions and, therefore, may be useful in their prevention. The effects of Meloxicam could not only be due to its anti-inflammatory action, but also to its effects on the expression of the Vascular Endothelial Growth Factor.
\end{abstract}

Keywords: Cyclooxygenase 2 Inhibitors; Experimental Animal Models; Ceneral Surgery; Non-Steroidal Anti-Inflammatory Agents; Tissue Adhesions.

About the Author: Luis Hernández is a doctor of medicine, recently graduated from the University of Oriente. He is winner of the second place in the international competition of research works in the XXVIII International Scientific Congress of the Latin American Federation of Scientific Societies of $\mathrm{Me}$ dical Students and winner of the first place in clinical cases and the third place in research works in the III Congress of Students of Medicine of the University of the Andes and Regional Course Zone $\mathrm{C}$ of the Latin American Federation of Scientific Societies of Medical Students.

\section{Introduction}

Peritoneal adhesions are pathological junctions of connective tissue formed between organs and tissues, and frequently between the omentum, intestines and abdominal wall. The etiology may be congenital or acquired. The acquired adhesions are classified postoperatively or postinflammatory. Inflammatory adhesions result from an inflammatory response of the peritoneum during an intra-abdominal inflammatory process, such as appendicitis and pelvic inflammatory disease. Post-surgical adhesions develop when a tissue is injured by surgical manipulation. ${ }^{1,2}$

In a prospective study, $93 \%$ of patients with a previous laparotomy had peritoneal adhesions, and the incidence of readmissions directly related to adhesions varies from $5 \%$ to $20 \%{ }^{3.4}$ Each year, 400,000 adhesiolysis procedures are performed in the United States, with a cost in the health system of close to $\$ 2$ trillion in hospitalizations and surgeries. ${ }^{5}$

Adhesions are the result of tissue trauma that may be the consequence of thermal or mechanical injury, infection, radiation, ischemia, dissection, abrasion, or foreign body reaction. 6 However, the most important and potential consequences resulting from the formation of peritoneal adhesions are: infertility, intestinal obstruction, and pelvic-abdominal pain. They can affect fertility by distorting the attached anatomy and interfere with the transport of the gamete and embryo. ${ }^{6}$
The most serious of the complications caused by adhesions is small bowel obstruction.? Adhesions account for $56 \%$ of postoperative intestinal obstructions.8 This surgical emergency has a mortality rate of $3-10 \%$ for simple obstruction, and up to $30 \%$ when the intestine is necrotic or perforated. In one study, it was evidenced that of 2000 patients submitted to laparotomy 1-2\% developed obstruction secondary to peritoneal adhesions in the same year of surgery. The incidence of intestinal obstruction secondary to adhesion formation is $1-10 \%$ in $4-6$ years after appendectomy, $6 \%$ in 5 years after cholecystectomy, $9-25 \%$ in 2-10 years following intestinal surgery, and $17-25 \%$ in 5-10 years following proctocolectomy. ${ }^{8}$

However, peritoneal adhesions are not a new problem, surgeons have studied different barrier/pharmacological agents to prevent the formation of adhesions. 9 In this way, different synthetic barrier methods have been used for its prevention. ${ }^{10.11}$ Among the pharmacological agents studied are: allopurinol, thymoquinone, phospholipids, spironolacton e, captopril, heparin has been studied. 1, 6, 12-14 Also a variety of steroids and anti-inflammatory agents have been studied, including aspirin, dexamethasone, methylprednisolone, estrogen, progesterone and budesonide. ${ }^{15}$ Likewise, the use of hemostatic agents, and the effects of vitamin $E$ and amniotic membrane on the formation of adhesions have been researched. ${ }^{16,17}$ However, none of these pharmacological agents and barrier methods have demonstrated clinically relevant results in reducing chronic pain, decreased infertility, and rate of reoperation. ${ }^{18}$

${ }^{1}$ University of Oriente 
Figure 1. Some of the Procedures Performed During the Adhesion-Forming Surgery and the Immediate Postoperative Period of the Rat. A. Exposure of the large intestine of the rat B. The skin and subcutaneous cellular tissue were closed with 4-o nylon. C. Postoperative immediately, the experimental animal is in an incubator (medix®, model PC-305) adjusted to 32ćC, enabled for its recovery.

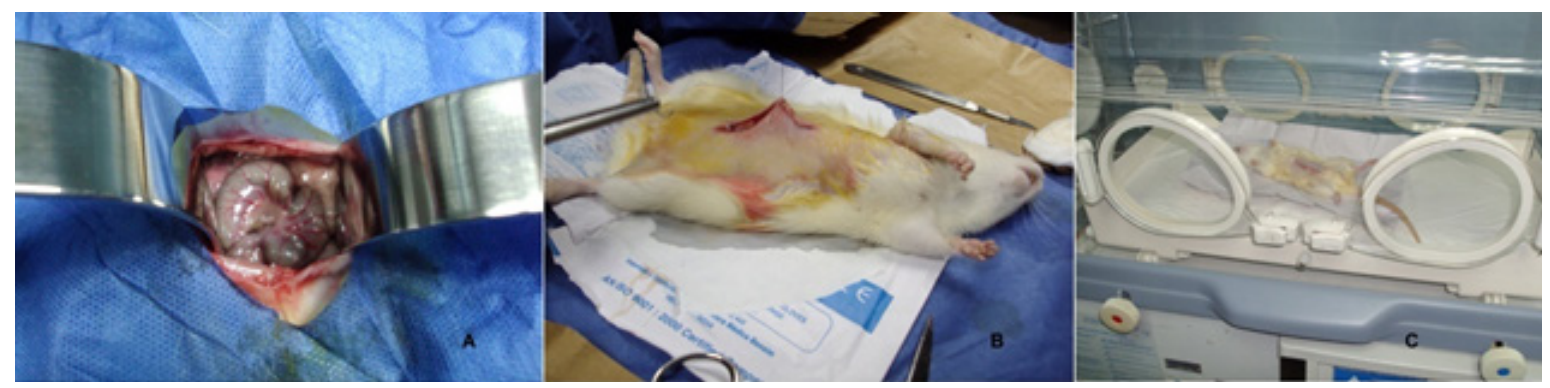

Meloxicam, is a non-steroidal anti-inflammatory categorized as a selective COX-2 inhibitor. It is commonly used in the treatment of acute and chronic pain and inflammation.19 The objective of this study is to evaluate the anti-inflammatory effects of Meloxicam in the formation of postoperative peritoneal adhesions in an experimental animal model. Therefore, in the present study we proposed the use of an experimental model of adhesion formation by serosal abrasion of the colon, having a control group without drug administration and a group to which Meloxicam was given intramuscularly. In light of the above considerations and by its mechanism of action, it is proposed as an alternative hypothesis that Meloxicam inhibits the formation of postoperative peritoneal adhesions; and as a null hypothesis, that Meloxicam does not inhibit the formation of adhesions.

\section{Materials and Methods}

An experimental study was carried out in an animal model, in which 20 female rats, Wistar strain, between 250 and $300 \mathrm{~g}$ in weight were used. Two experimental rats were kept per cage, with food and water to free demand. The bed of each cage was changed twice a week. They were under cycles of 12 hours light and 12 hours darkness and a temperature in the experimental laboratory of $22 \dot{c} \mathrm{C} \pm 2 \dot{c} \mathrm{C}$.

All procedures of the research protocol were carried out strictly taking into account the principles for the care and use of laboratory animals, according to the bioethics criteria for the experimentation of the Venezuelan Association for the Science of Laboratory Animals.20

Before the operation, the experimental animals were randomly distributed into two groups consisting of 10 animals each: a control group and a study group.

In each experimental rat, we proceeded to perform a surgical procedure with the goal to induce the adhesion formation process. Each subject was previously anesthetized by administration of $100 \mathrm{mg} / \mathrm{kg}$ Ketamine (Keiran $®$ ) and $10 \mathrm{mg} / \mathrm{kg}$ Xylazine (Rompun $®$ ), both intramuscularly.

The abdominal skin was disinfected with Povidone Iodine solution (BETADINE $®$ ), prior to the procedure. After, a vertical midline incision measuring $3 \mathrm{~cm}$ in length was made, the large intestine of the rat was exposed and the induction of the process of formation of peritoneal adhesions was carried out by a technique already described, which consisted of injuring the serosa of the large intestine by vigorous rubbing with dry gauze (Figure $1 \mathrm{~A}$ ). The rubbing was maintained until the appearance of hemorrhagic points. This procedure was performed in 5 segments corresponding to the cecum, 1 ascending colon segment, 1 transverse colon segment, 1 descending colon segment and sigmoid segment. Each segment was $1 \mathrm{~cm}$ in length.

The abdominal wall was closed, suturing the aponeurosis and rectus abdominis muscle with Polyglactin 910 (Vycril@) 4-0; skin and subcutaneous cellular tissue with 4-0 nylon (Figure $1 B)$. At the end of the surgical procedure, each experimental animal was placed in an incubator (Medix $®$, model PC-305) set at $32 c \mathrm{C}$, where they lasted for 4 hours with the finality to achieve recovery (Figure $1 \mathrm{C}$ ).

In terms of treatment, the control group had no therapy applied. The study group, was administered Meloxicam (Mobic $($, Boehringer Ingelheim) intramuscularly, at a dose of 0.20 $\mathrm{mg} / \mathrm{kg} /$ day, for 7 days, starting right after the surgery.

All experimental rats were sacrificed at day 15 postoperative, under the effects of anesthesia already described. $1 \mathrm{~mL}$ of $7.5 \%$ Potassium Chloride was administered via intracardiac injection, via a thoracotomy to achieve exposure of the heart.

An abdominal " $U$ " incision was made by lifting the abdominal wall of the experimental rat with the objective to evaluate the adhesions formed, registering their presence or absence, formation in unmanipulated organs, and whether there were anterior or posterior abdominal wall attachments. Likewise, each of the adhesions presented by the experimental animals of each group was counted.

The degree of severity, dissection and extension were evaluated according to the classification of Diamond.21 According to the severity, it was considered: Grade 0, without adhesions; Grade 1, thin and avascular adhesions were evident; Grade 2, vascularized and dense adhesions were observed; Grade 3, adhesions were firm and cohesive. As for its extension: Grade 0 , without adhesions; Grade 1, less than 25\%; Grade 2, between $26 \%$ and $50 \%$, Grade 3, more than $50 \%$ of surface. The density was evaluated by the following: Grade 0 , without adhesions; Grade 1, the adhesions were released spontaneously upon se- 
Figure 2. Degree of severity, density and extent of peritoneal adhesions. *Statistical significance compared with control group.

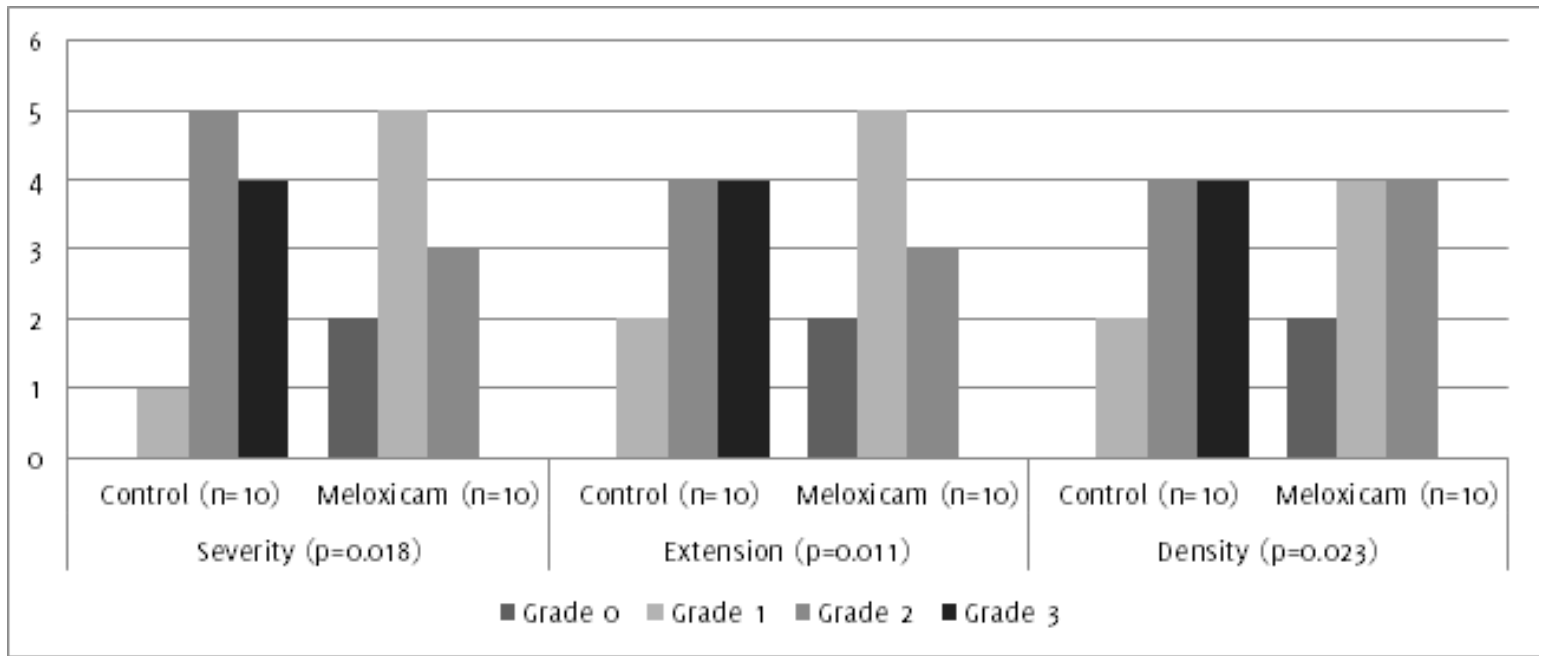

paration of the flap; Grade 2, mild to moderate traction was required to separate the adhesions; Grade 3, those that merited adhesiolysis with scissors. Adhesion tissue samples were dehydrated, placed in paraffin, and then 3 micron cuts were made with a rotating microtome (MICROM $®)$. The sections were stained with Haematoxylin and Eosin (H/E) and Masson's Trichrome stain. The surgical pieces were analyzed by a blind observer to the procedure, in the laboratory of Histopathology of the Hospital Complex University Ruíz and Paez, Ciudad Bolívar, Venezuela.

The histological characteristics of the adhesions were determined according to the histological classification of Kanbour-Shakir, which evaluates three aspects: fibrosis, inflammation and vascular proliferation. Firstly, fibrosis is measured by the percentage of occupation of fibroblasts in a dry field of observation (mild $<33 \%$, moderate $>33 \%$ and $<66 \%$, severe $>66 \%$, and no fibroblast proliferation). Secondly, inflammation involves observing the presence of inflammatory cells and classifying it as mild (infiltrating with occasional giant cells, lymphocytes and plasma cells), moderate (giant cells, eosinophils and neutrophils) and severe (abundant inflammatory cells and microabscesses) or absence of an inflammatory component. Lastly, vascular proliferation, defined as the number of blood vessels present per area in 40x magnification or high powered field, classified as mild (blood vessels in less than $33 \%$ of the field extension), moderate (in more than $33 \%$ and less than $66 \%$ of the area) and severe (> $66 \%$ ) or without blood vessels. 22

Statistical analysis was performed using the SPSS software (version 23; Statistical Package for the Social Sciences, SPSS Inc, Chicago, IL). The normality of the variables was evaluated by the Shapiro-Wilk test. For the comparison of the means of each group: the quantitative variables with a normal distribution were assessed with the Student's t-test for independent samples; variables not fulfilling a normal distribution were evaluated by the Mann-Whitney U-test; and the qualitative variables were evaluated with the Chi-square test. The confidence interval was set at $95 \%$ and the differences were considered statistically significant when the $p$-value was, 0.05 .

\section{Results}

All experimental animals completed the study. No congenital adhesions were evident in any of the rats in the first surgical intervention. After the surgical procedure, there were no complications such as wound infections, peritonitis or intestinal obstruction.

Regarding the presence of adhesions formed after the surgical in-

Figure 3. Degrees of Severity of Peritoneal Adhesions Evidenced During Macroscopic Assessment. A. Rat of the study group (Meloxicam), which demonstrates a loose adhesion that could be released exclusively with traction. B. Strong and cohesive peritoneal adhesions between several thin intestinal loops, with a greater than $50 \%$ extension of the abdominal cavity, which required adhesiolysis with scissors for their release. C. Adhesions of the small intestine to the anterior abdominal wall (in the control group rat) involving more than $50 \%$ of the abdominal cavity with a high degree of severity and impossibility of release, only by adhesiolysis.
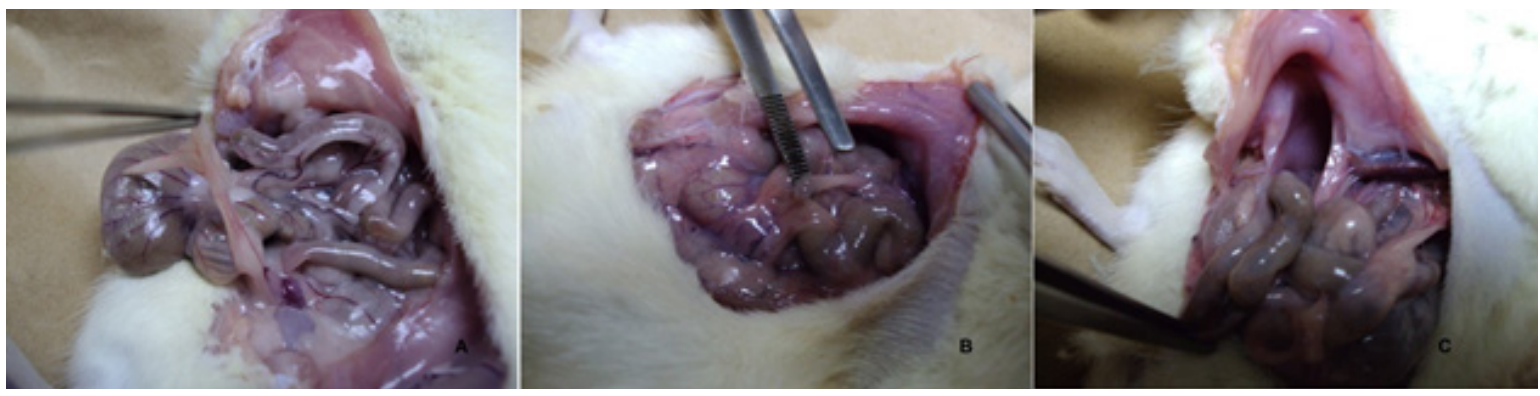
duction procedure, 18 of 20 research animals had at least one adhesion. Peritoneal adhesions were formed in all rats (10 of 10 animals) belonging to the control group, while in the rats belonging to the study group (with administration of Meloxicam) the adhesions were evident in 8 of 10 experimental animals.

It was observed that the group of animals that were administered meloxicam after the surgical procedure two animals had only a single adhesion. The total adhesions per experimental group were: 32 in the group treated with meloxicam and 183 in the control group. The mean number of adhesions per group was 18.3 for the control group and 3.2 for the study group (Table 1). These differences were statistically significant (control vs. study group, $p=0.018)$.

7 of 10 animals in the control group presented with adhesions in unmanipulated organs during the surgical procedure, whereas in the study group none of the experimental animals presented with adhesions in unmanipulated organs. There was a statistically significant difference between the two groups, $\mathrm{p}=0.001$ (Table 2). The presence of adhesions with anterior or posterior abdominal wall attachment occurred in 4 of 10 animals belonging to the control group. However, this characteristic was not evidenced in the group of animals who were administered meloxicam. There was a statistical difference denoting $p=0.025$, in relation to adhesions to the anterior and posterior abdominal wall (Tables 3 \& 4 ).

Regarding the assessment of adhesions, statistically significant differences were evidenced, according to their severity, extension and density (ease for dissection), with corresponding $p$ values: $p=$ $0.004, p=0.011$ and $p=0.023$, respectively (Figures 2 it 3 ).

In the histopathological study (Figures 4 it 5), the development of different degrees of fibrosis was evidenced, depending on the treatment group. Animals treated with Meloxicam had a lower degree of fibrosis, and this difference was statistically significant when compared to the control group $(p=0.029)$.

Likewise, a statistically significant difference was observed when the inflammation and vascular proliferation were evaluated in both experimental groups, denoting $p$ values of $p=0.002$ and $p=$ 0.004 , respectively.

Figure 4. Degrees of fibrosis, inflammation and vascular proliferation in postoperative peritoneal adhesions. *Statistical significance compared with control group.

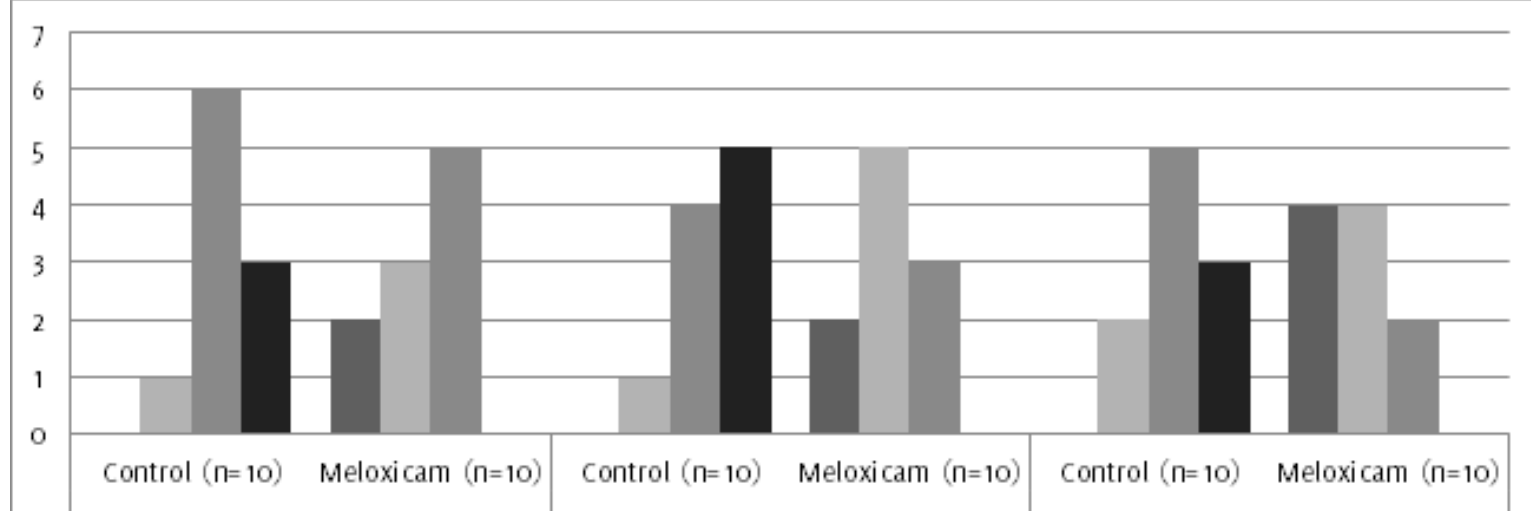

\section{Discussion}

The objective of this study was to evaluate the effects of Meloxicam on the formation of postoperative peritoneal adhesions in an experimental animal model, which consisted of the serous abrasion of the rat colon. The results of the macroscopic evaluation showed that the animals belonging to the group administered Meloxicam developed fewer adhesions and had no adhesions in unmanipulated organs. As for the histological evaluation, it was evidenced that the Meloxicam group under study developed a lower degree of fibrosis, inflammation and vascular proliferation. In contrast, after peritoneal trauma by serosal abrasion of the colon, all rats in the control group developed peritoneal adhesions and greater degrees of severity than the rats given Meloxicam.

The trauma to the peritoneum triggers a cascade of events that begins with the disruption of mast cells, which release vasoactive substances such as histamine that increase vascular permeability. 6 In addition, extravasation of a fibrinogen rich fluid occurs from the injured surfaces. Simultaneously, an inflammatory response occurs, with migration of inflammatory cells, release of cytokines and activation of the coagulation cascade. Activation of the coa- gulation system results in the formation of thrombin, which is necessary for the conversion of fibrinogen to fibrin. Since fibrinolysis is the key determinant in the formation of adhesions. If this does not occur within 5 to 7 days following peritoneal injury, the fibrin matrix persists and is gradually further organized with collagen secreting fibroblasts. ${ }^{4}$

Fibroblasts and myofibroblasts secrete massive amounts of extracellular matrix molecules including fibronectin, hyaluronic acid, glycosaminoglycans, and proteoglycans. This process establishes a bridge between tissues within a few weeks. Further evidence includes vascularization and deposits of collagen in this adhesion bridge formed between the two tissues. ${ }^{9}$

The formation of peritoneal adhesions results from a complex cascade regulated by different cellular and humoral factors. Among the cellular factors are the mesothelial cells, different types of inflammatory cells and fibroblasts. The relationship of these cells and their structural organization is regulated by cytokines, growth factors and signaling molecules. It is widely accepted that in local tissue injury, ischemia, the resulting inflammatory response, and 
Figure 5. Images Obtained from the Histopathological Evaluation of Experimental Animals. A. Histopathological image representing a sample of tissue with adhesions in which large areas of loose connective tissue and inflammatory infiltrate (star), with presence of giant cells (green arrow), fibroblast (blue arrow) and mild vascular proliferation (black arrow) were observed (Hematoxylin-eosin stain; original magnification $\mathrm{X}_{40}$ ). B. Sample of tissue in which the intestinal mucosa (star), the muscularis externa (blue arrow), areas of fibrosis and presence of infiltration of inflammatory cells (black arrow) were observed (Hematoxylin-eosin stain; original magnification $\mathrm{X}_{10}$ ) C. Sample of tissue in which were evidenced: the intestinal mucosa (star), the muscularis externa (blue arrow) and areas of loose connective tissue (black arrow). (Hematoxylin-eosin stain; original magnification $\mathrm{X}_{5}$ ). D. Histological image belonging to rat, in which no adhesions were observed in the macroscopic evaluation. Highlighted areas include the intestinal mucosa (star), the submucosa (black arrow) and the muscularis externa (blue arrow). (Hematoxylin-eosin stain; original magnification $\mathrm{X}_{5}$ ). E. Histological image in which the intestinal mucosa (black star), the submucosa (blue star) with blood vessels (black arrow), the muscularis externa (blue arrow) and the serosa (green arrow), without inflammatory process, vascular proliferation and fibrosis. (Hematoxylin-eosin stain; original magnification $\mathrm{X}_{10}$ ).

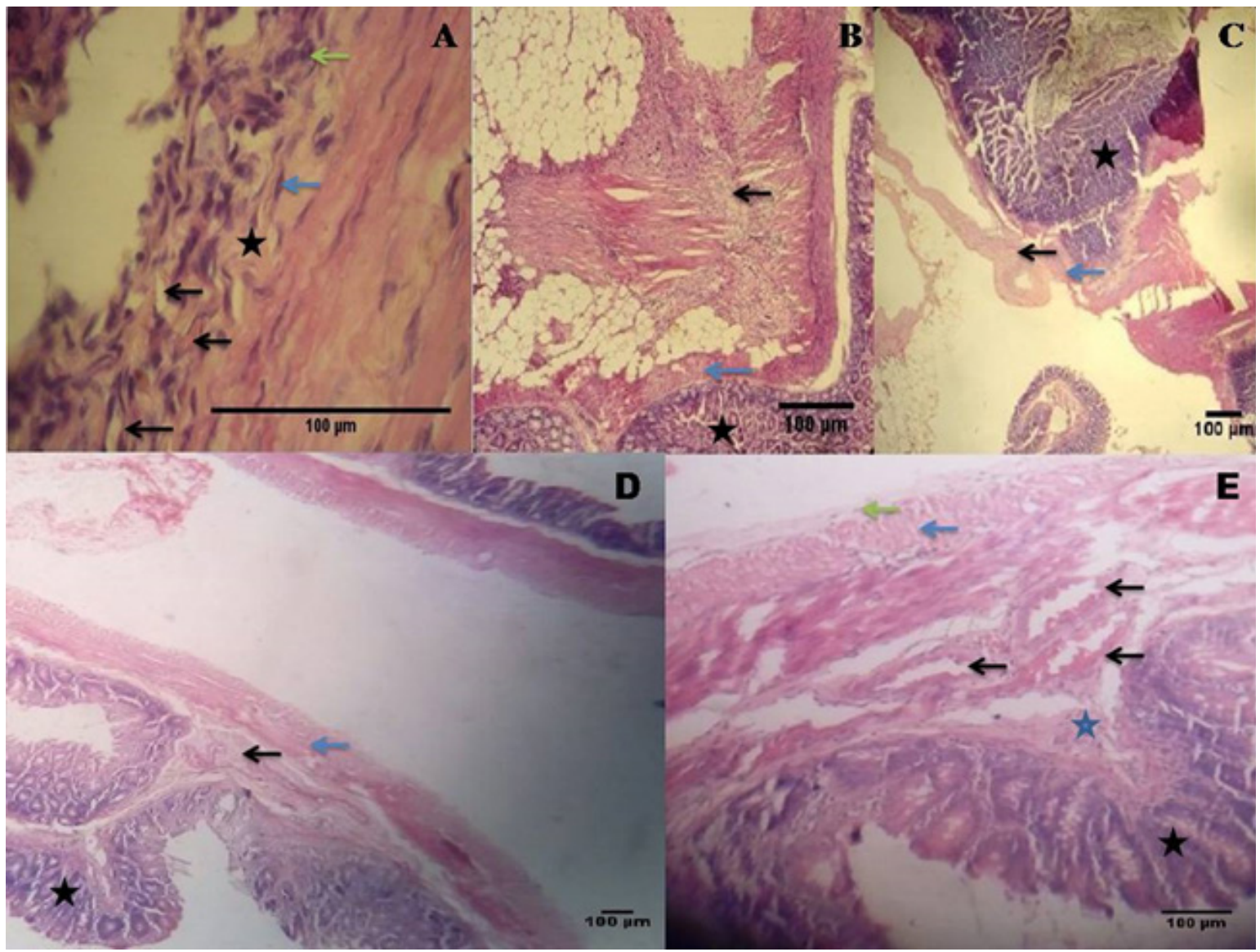

the promotion of procoagulatory processes such as antifibrinolytic reactions are essential for the formation of peritoneal adhesions. ${ }^{23}$

Peritoneal damage causes an inflammatory response, in which inflammatory cells release cytokines, such as Tumor Necrosis Factor alpha (TNF-alpha), interleukin 1 and 6. These cytokines induce the release of plasminogen activator inhibitor- 1 and 2 (PAI- 1 and PAl-2) of mesothelial cells, which results in a reduction in Plasminogen activator (Pas) activity. In this way, PAl inhibits fibrinolysis, and fibrin deposits are infiltrated by granulocytes, monocytes and fibroblasts, followed by capillary growth, collagen deposits and adhesion formation. ${ }^{24}$

Likewise, an increase in COX-2 expression has been shown in response to hypoxia in normal peritoneum fibroblasts. However, COX-1 expression remains unchanged in adhesions and fibroblasts under conditions of normoxia and hypoxia. It is hypothesized that hypoxia leads to fibroblasts of the normal peritoneum to acquire a phenotype of adhesions as a manifestation of the marked increase in the expression of COX-2. 25 Hypoxia induces normal peritoneal fibroblasts to produce high levels of PGE-2 and COX-2, an effect that can be prevented by inhibition of COX-2. Therefore, it is considered that COX-2 and its inhibitor may play a role in the postoperative regulation of tissue repair and the development of adhesions. ${ }^{26,27}$

VEGF is an angiogenic cytokine that participates in the adhesion formation process through the formation of new vessels. It is implicated in early inflammatory responses, tissue repair and remodeling through fibroblast function. It is also important to facilitate fibrin rich matrix deposition, necessary for cell migration and proliferation. ${ }^{28,29}$

Also, it has been suggested that laparotomy can stimulate the for- 
mation of adhesions through a cellular process dependent on mast cells, an inflammatory process that is independent of immediate degranulation. Mast cells are probably not responsible for all locally released VEGF, because this cell produces cytokines that induce the influx of other inflammatory cells that could produce VEGF at the site of the lesion. ${ }^{28,29}$

It has been shown that dexamethasone, a steroidal anti-inflammatory, in combination with sodium carboxymethylcellulose can prevent the formation of adhesions in a rat adhesion-forming model, inhibiting the migration of inflammatory cells, further decreasing the proliferation of fibroblasts..$^{30}$

Likewise, the use of non-steroidal anti-inflammatory drugs, such as Diclofenac Sodium, has been effective in decreasing adhesion formation in a model of anti-mesenteric border lesion in the rat coIon. In this same study, there was a decrease in the development of edema, hyperemia, inflammation and fibrosis. ${ }^{31}$

Similarly, inhibition of COX-2 by the administration of parecoxib, celecoxib, rofecoxib and nimesulide has been shown to decrease adhesion formation in animal models. ${ }^{32,33,34,35}$ Guvenal et al. associated the effects of nimesulide in its study to its anti-prostaglandin activity and the reduction of the production of anti-angiogenic

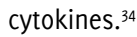

However, in a study by Keskin et al., where Meloxicam and Dexketoprofen were evaluated in a rat uterine horn surgical model, it was shown that Meloxicam decreased the development of inflammation. However, despite a decrease in vascular proliferation and collagen formation, there was no significant decrease in adhesion formation.36 In contrast, in the present study Meloxicam was shown to decrease the formation of adhesions, the development of fibrosis, vascular proliferation and inflammation. This may be due to the fact that different techniques and experimental models were used in both studies. In addition, in the same study, Meloxicam was administered 2 days before surgery and 5 days after surgery, whereas in this study meloxicam was given for 7 days after the surgical procedure.

In an experimental model of periodontitis in rats, it was shown that after 14 days of treatment with Meloxicam, there was a decrease in the expression of VEGF expression.37 Similarly, it has been shown that Meloxicam decreases VEGF levels in tumor tissues from animal experimental models. 38 In a model of ovarian hyperstimulation syndrome in rats, the results suggest that Meloxicam affects the expression of VEGF in the ovary. 39

In addition to the inhibition of the inflammatory process involved in the formation of adhesions in the Meloxicam group, there was also a significant decrease in the development of vascular proliferation. The mechanism is most likely due to a decrease in the production of VECF by the cells involved in the process of adhesionogenesis, such as mast cells.

Some limitations were evident in our study. The pathophysiological process of peritoneal scarring was one of the limitations, because the mechanisms of adhesion formation in human have not been studied in great detail to warrant a direct comparison to the effects of meloxicam on adhesion formation in rats. The adverse and side effects of Meloxicam was also not examined in the study at the dose $(0.20 \mathrm{mg} / \mathrm{kg} /$ day) and duration of treatment (7 days) used in the study. Despite the limitations of the study, meloxicam was shown to decrease the formation of postoperative peritoneal adhesions in the experimental model used.

In conclusion, Meloxicam, proved to be effective in the prevention of post-surgical peritoneal adhesions induced in the animal model used. It is a promising finding, based on the pathophysiological knowledge of inflammation, peritoneal healing and the involvement of VEGF in the formation of peritoneal adhesions. Therefore, it is proposed to continue the research of this drug in other models of experimentation, while performing the quantification of inflammatory markers, cytokines in plasma and peritoneal fluid. The final objective of future studies will be to understand the effects, positive or negative, of Meloxicam in the formation of adhesions.

Table 1. Clinical outcomes when comparing the interventions.

\begin{tabular}{|c|c|c|c|}
\hline Characteristic & Control $(n=10)$ & Meloxicam $(n=10)$ & P-value \\
\hline Peritoneal adhesions, mean (SD) & $18.30(16.45)$ & $3.20(2.15)$ & 0.018 \\
\hline $\begin{array}{l}\text { Peritoneal adhesions formed in non-manipula- } \\
\text { ted organs* }\end{array}$ & & & 0.003 \\
\hline Yes, $n(\%)$ & $7(100)$ & $0(0)$ & 0.003 \\
\hline No, $n(\%)$ & $3(23.08)$ & $10(76.92)$ & 0.003 \\
\hline $\begin{array}{l}\text { Peritoneal adhesions formed attached to } \\
\text { anterior abdominal wall* }\end{array}$ & & & 0.087 \\
\hline Yes, $n(\%)$ & $4(100)$ & $0(0)$ & 0.087 \\
\hline No, $n(\%)$ & $6(37.50)$ & $10(62.50)$ & 0.087 \\
\hline $\begin{array}{l}\text { Peritoneal adhesions formed attached to posterior } \\
\text { abdominal wall* }\end{array}$ & & & 0.087 \\
\hline Yes, $\mathrm{n}(\%)$ & $4(100)$ & $0(0)$ & 0.087 \\
\hline No, $n(\%)$ & $6(37.50)$ & $10(62.50)$ & 0.087 \\
\hline
\end{tabular}




\section{References}

1. Bozdag Z, Gumus M, Arikanoglu Z, Ibiloglu I, Kaya S, Evliyaoglu O. Effect of intraperitoneal Thymoquinone on Postoperative Peritoneal Adhesions. Acta Chir Belg. 2015(5); 115: 364-368.

2. De Clerq K, Sheltfhout C, Bracke M, De Wever 0, Van Bockstal M, Ceelen $W$, et al. Cenipin-crosslinked gelatin microspheres as a strategy to prevent postsurgical peritoneal adhesions: In vitro and in vivo characterization. Biomaterials. 2016; 96: 33-46.

3. Szomstein S, Lo Menzo E, Simpfendorfer C, Zundel N, Rosenthal RJ. Laparoscopic Lysis of Adhesions. World J Surg. 2006; 30(4): 535-40.

4. Schnuriger B, Barmparas G, Branco BC, Lustenberger T, Inaba K, Demetriades $D$. Prevention of postoperative peritoneal adhesions: a review of the literature. Am J Surg. 2011; 201(1): 111-121.

5. Dubcenco E, Assumpcao L, Dray X, Gabrielson KL, Ruben DS, Pipitone LJ, et al. Adhesion formation after peritoneoscopy with liver biopsy in a survival porcine model: comparison of laparotomy, laparoscopy, and transgastric natural orifice transluminal endoscopic surgery (NOTES). Endoscopy. 2009; 41(11): 971-978.

6. Practice Committee of American Society for Reproductive Medicine in coIlaboration with Society of Reproductive Surgeons. Pathogenesis, consequences, and control of peritoneal adhesions in gynecologic surgery: a committee opinion. Fertil Steril. 2013; 99(6): 1550-1555.

7. Rajab TK, Wallwiener M, Talukdar S, Kraemer B. Adhesion-Related Complications Are Common, But Rarely Discussed in Preoperative Consent: A Multicenter Study. World J Surg. 2009; 33(4): 748-750.

8. ten Broek RP, Issa Y, van Santbrink EJ, Bouvy ND, Kruitwagen RF, Jeeker J, et al. Burden of adhesions in abdominal and pelvic surgery: systematic review and met-analysis. BMJ. 2013; 347: f5588.

9. Ward BC, Panitch A. Abdominal Adhesion: Current and Novel Therapies. J Surg Res. 2011; 165(1): 91-111.

10. Ahmad C, Duffy JM, Farquhar C, Vail A, Vandekerchove P, Watson A, et al. Barrier agents for adhesion prevention after gyneacological surgery (Review). Cochrane Database of Syst Rev. 2008;(2):CDoo0475.

11. Wallwiener M, Brucker S, Hierlemann H, Brochhausen C, Solomayer E, Wallwiener $C$. Innovative barriers for peritoneal adhesion prevention: liquid or solid? A rat uterine horn model. Fertil Steril. 2006; 86 (4 Suppl): 1266-1276. 12. Ayala M, Ramírez E, Quiroz ], Ortiz ], Conzález B. [Role of alopurinol in peritoneal adherences when placing a polypropylene mesh: Experimental study]. Cir Gen. 2013; 35(1): 16-19.

13. Fotiadis K, Filidou E, Arvanitidis K, Valatas V, Stavrou G, Basdanis G, et al. Intraperitoneal application of phospholipids for the prevention of postoperative adhesions: a possible role of myofibroblasts. J Surg Res. 2015;197(2): 291-300.

14. Vázques CJ, Ortiz MM, Sánchez CJR, Reynoso VJ, Gutiérrez I, Gutiérrez C. [Decrease of angiogenesis with spironolactone and captopril and the effect on intraperitoneal adherences]. Cir Gen. 2007; 29(4): 265-268. Spanish.

15. Maciver AH, McCall M, James Shapiro AM. Intra-abdominal adhesions: cellular mechanisms and strategies for prevention. Int J Surg. 2011; 9(8): 589-594.

16. Hoffmann NE, Siddiqui SA, Agarwal S, McKellar SH, Kurtz HJ, Gettman MT, et al. Choice of Hemostatic Agent Influences Adhesion Formation in a Rat Cecal Adhesion Model. J Surg Res. 2009; 155(1): 77-81.

17. Yetkin G, Uludag M, Citgez B, Karakoc S, Polat N, Kabukcuoglu F. Prevention of peritoneal adhesions by intraperitoneal administration of vitamin $E$ and human amniotic membrane. Int J Surg. 2009; 7(6): 561-565.

18. Koninckx P, Corona R, Timmerman D, Verguts J, Adamyan L. Peritoneal full-conditioning reduces postoperative adhesions and pain: a randomised controlled trial in deep endometriosis surgery. J Ovarian Res. 2013; 6(1):90. 19. Huang LN, Yao XM. Inhibitory effect of Meloxicam on the cultured fibroblasts from the excised pterygium. Int J Ophthalmol. 2008; 1(1): 48-51.
20. AVECAL (Venezuelan Association for the Science of Laboratory Animals). [Manual for the production and ethical use of laboratory animals]. Ministry of Popular Power for Science and Technology. Caracas. 2008. Spanish.

21. Mayagoitia-Gonzalez JC, Gudiño-Amezcua LM, Rivera-Barragan V, Mellado-Diaz AV, Díaz-Chavez EP. [Prevention of intestinal adhesions, through the addition of hyaluronic acid/carboxymethylcellulose gel. Experimental model in rats]. Cir Cir. 2012; 80(2): 150-156.

22. Marentes Etienne JJ, Joya Cervera RE, Rodríguez Hernandez A, Díaz-Chavez EP. [Efficacy of a silicone composite mesh to reduce intra-abdominal adhesions in wistar rats: A preliminary report]. Cir Cen. 2014; 36(4): 205-208. Spanish.

23. Brochhausen C, Schmitt VH, Planck CN, Rajab TK, Hollemann D, Tapprich C, et al. Current strategies and future perspectives for intraperitoneal adhesion prevention. J Gastrointest Surg. 2012; 16(6): 1256-1274.

24. Fredriksson F, Christofferson RH, Carlsson PO, Lilja HE. Locally increased concentrations of inflammatory cytokines in an experimental intrabdominal adhesion model. J Pediatr Surg. 2014; 49(10): 1480-1484.

25. Braun KM, Diamond MP. The Biology of Adhesion Formation in the Peritoneal Cavity. Semin Pediatr Surg. 2014; 23(6): 336-343.

26. Saed GM, Munkarah AR, Diamond MP. Cycloxygenase-2 is expressed in human fibroblasts isolated from intraperitoneal adhesions but not from normal peritoneal tissues. Fertil Steril. 2003; 79(6): 1404-1408.

27. Saed GM, Munkarah AR, Abu-Soud HM, Diamond MP. Hypoxia upregulates cycloxygenase-2 and prostaglandin E2 levels in human peritoneal fibroblasts. Fertil Steril. 2005; 83 Suppl 1: 1216-1219.

28. Cahill RA, Wang JH, Soohkai S, Redmond HP. Mast cells facilitate local VEGF release as an early event in the pathogenesis of postoperative peritoneal adhesions. Surgery. 2006; 140(1): 108-112.

29. Cahill RA, Redmond HP. Cytokine orchestration in post-operative peritoneal adhesion formation. World J Gastroenterol. 2008; 14(31): 4861-4866.

30. Du XH, Liu JQ, Xin K, Liu GH. Dexamethasone and sodium carboxymethyl cellulose prevent postoperative intraperitoneal adhesions in rats. Braz J Med Biol Res. 2015; 48(4): 344-348.

31. Allahverdi TD, Allaverdi E, Yayla S, Deprem T, Merhan O, Vural S. The Comparison of the Effects of Ellagic Acid and Diclofenac Sodium on Intra-Abdominal Adhesion: An In Vivo Study in the Rat Model. Int Surg. 2014; 99(5): 543-550.

32. Arung W, Jehaes F, Cheramy JP, Defraigne J0, Meurisse M, Honore $P$, et al. Effects of Parecoxib on The Prevention of Postoperative Peritoneal Adhesions in Rats. J Invest Surg. 2013; 26(6): 340-346.

33. Greene AK, Alwayn IP, Nose V, Flynn E, Sampson D, Zurakowski D, et al. Prevention of Intra-abdominal Adhesions Using the Antiangiogenic COX-2 inhibitor Celecoxib. Ann Surg. 2005; 242(1): 140-146.

34. Guvenal T, Cetin A, Ozdemir H, Yanar 0, Kaya T. Prevention of postoperative adhesión formation in rat uterine horn model by nimesulide: a selective COX-2 inhibitor. Hum Reprod. 2001;16(8): 1732-1735.

35. Guvenal T, Yanar 0, Timuroglu Y, Cetin M, Cetin A. Effects of selective and non-selective cyclooxygenase (COX) inhibitors on postoperative adhesión formation in a rat uterine horn model. Clin Exp Obstet Cynecol. 2010; 37(1): 49-52.

36. Keskin HL, Akkus SM, Sirens YS, Ustuner I, Keles H, Ide T, et al. Comparison of the Effects of Meloxicam and Dexketoprofen on Postoperative Adhesion Formation in a Rat Uterine Horn Surgical Model. J Minim Invasive Cynecol. 2013; 20(2): 185-191.

37. Oliveira TM, Sakai VT, Machado MA, Dionísio TJ, Cestari TM, Taga R, et al. COX-2 Inhibition Decreases VEGF Expression and Alveolar Bone loss During the Progression of Experimental Periodontitis in Rats. J Periodontol. 2008; 79(6): 1062-1069.

38. Xin B, Yokoyama Y, Shigeto T, Futagami M, Mizunuma H. Inhibitory Effects of Meloxicam, a Selective Cyclooxygenase-2 inhibitor, and Ciglitazone, a Peroxisome Proliferator-Activated Receptor Gamma Ligand, on the Growth of 


\section{Original Article}

Human Ovarian Cancers. Cancer. 2007; 110(4): 791-800.

39. Quintana R, Kopcow L, Marconi G, Young E, Yovanovich C, Paz DA. Inhibi-

tion of cyclooxygenase-2 (COX-2) by meloxicam decreases the incidence of ovarian hyperstimulation syndrome in a rat model. Fertil Steril. 2008; 90 (4 Suppl): 1511-1516.

\section{Acknowledgments}

Special acknowledgments go to the School of Health Sciences, "Dr. Francisco Battistini Casalta", and the University of Oriente.

Conflict of Interest Statement ct Funding

None of the authors shows any conflicts of interest during the investigation or publication of this article.

Author Contributions

Conception and design the work/idea, Write de manuscript, Contribution of patients or study material, Statistical advice: LAHV. Collect data / obtaining results: LAHV, HF. Analysis and interpretation of data, Critical revision of the manuscript, Approval of the final version: LAHV, HF, LC.

Cite as:

Hernandez-Villarroel LA, Fernandez H, Cesin L. Meloxicam Decreases the Formation of Peritoneal Adhesions in an Experimental Surgical Model in Rats. Int J Med Students. 2017 Jan-Apr;5(1):6-13. 